\title{
Asociaciones longitudinales entre el rasgo de mindfulness y conductas adictivas en adolescentes
}

\section{Longitudinal associations between dispositional mindfulness and addictive behaviors in adolescents}

\author{
Nerea Cortazar*, Esther Calvete*. \\ * Universidad de Deusto. Departamento de Personalidad, Evaluación y Tratamientos Psicológicos, Bilbao, España.
}

\section{Resumen}

La adolescencia es un período vulnerable para el desarrollo de conductas adictivas. El uso de sustancias (US) y el uso problemático de Internet (UPI) generalmente comienzan durante esta etapa de desarrollo. El mindfulness rasgo (MD) se ha propuesto como un factor protector para los y las adolescentes frente a numerosos problemas psicológicos. Estudios previos sugieren que la faceta Observar de MD puede moderar los roles de las otras facetas. El objetivo del presente estudio fue analizar longitudinalmente si las facetas de MD podían predecir niveles más bajos de UPI y US entre los y las adolescentes, y evaluar si la faceta Observar moderaba las asociaciones entre las otras facetas de MD y las conductas adictivas. Un total de 836 participantes de entre 11 y 18 años completaron medidas de UPI, US y las cinco facetas de MD. Los resultados indicaron que Actuar con conciencia predijo niveles más bajos de US, Describir predijo un aumento tanto de UPI como de US y No juzgar predijo marginalmente niveles más bajos de UPI. Además, la faceta Observar fue beneficiosa frente a UPI cuando se combinó con altos niveles de Actuar con conciencia, pero no fue beneficiosa cuando se combinó con altos niveles de Describir. Se discuten las implicaciones y direcciones futuras para el estudio empírico de MD frente a conductas adictivas.

Palabras clave: Rasgo de mindfulness; uso problemático de Internet; uso de sustancias; adolescentes.

\section{Abstract}

Adolescence is a vulnerable period for the development of addictive behaviors, and substance use (SU) and problematic Internet use (PIU) typically start during this developmental stage. Dispositional Mindfulness (DM) has been proposed as a protective factor for adolescents against numerous psychological problems. Previous studies have suggested that the Observing facet of DM may moderate the other facets' roles. The objective of this study was to longitudinally analyze whether the facets of DM could predict lower levels of PIU and SU among adolescents, and to assess whether the Observing facet moderated the associations between the other facets of DM and addictive behaviors. A total of 836 participants aged 11 to 18 completed measures of PIU, SU, and the five facets of DM. The results indicated that Acting with Awareness predicted lower SU, Describing predicted an increase in both PIU and SU, and Non-judging marginally predicted lower PIU. Furthermore, Observing was beneficial against PIU when combined with high levels of Acting with Awareness, but was not when combined with high levels of Describing. The implications and future directions for the empirical study of DM against addictive behaviors are discussed.

Keywords: Dispositional mindfulness; problematic Internet use; substance use; adolescents. 


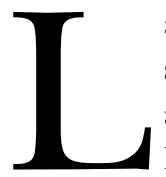

a adolescencia se considera una etapa del desarrollo caracterizada por numerosos cambios a nivel biológico, social, cognitivo y afectivo. Estos cambios hacen de esta etapa un período vulnerable para que los y las adolescentes desarrollen mayores niveles de conducta adictiva, ya que el consumo de sustancias (US) normalmente comienza en la adolescencia (Bava y Tapert, 2010). La edad promedio a la que el US comienza a mostrar una mayor prevalencia es de 14 años, siendo típico a esta edad el primer consumo de alcohol, y el primer consumo de otras sustancias ilegales, como cannabis y cocaína, a los 15 años (Observatorio Español de las Drogas y las Adicciones, 2019). Además, en los últimos años, con la expansión del uso de Internet entre la juventud, ha surgido la adicción a Internet o el uso problemático de Internet (UPI). El UPI se caracteriza por comportamientos asociados al control deficiente, el uso continuo y la preocupación cognitiva con respecto a Internet, que pueden acarrear una serie de consecuencias negativas en distintas áreas de la vida personal (Caplan, 2010). Los resultados de un estudio realizado con una gran muestra de adolescentes españoles/as indicaron una alta prevalencia de UPI, alcanzando el 16,3 \% (Gómez, Rial, Braña, Golpe y Varela, 2017). A su vez, estas conductas adictivas están relacionadas con niveles más altos de problemas de salud psicológica y física (Brownlie et al., 2019; Restrepo et al., 2020). Además, diferentes estudios indican que el UPI y el US están relacionados entre sí (Gámez-Guadix, Orue, Smith y Calvete, 2013b).

Considerando las tasas de ambas conductas de riesgo y su aparición precoz en la adolescencia, es necesario identificar factores protectores que ayuden a prevenir el desarrollo de estos problemas. Recientemente, ha aumentado el interés en las intervenciones basadas en mindfulness, siendo el Mindfulness Disposicional (MD) un factor beneficioso para prevenir el desarrollo de numerosos problemas psicológicos en diferentes poblaciones, incluidos los y las adolescentes.

\section{Mindfulness disposicional, uso de sustancias y uso problemático de Internet}

El MD se ha definido como un constructo multidimensional (p. ej., Baer, Smith, Hopkins, Krietemeyer y Toney, 2006; Bishop et al., 2004). Baer et al. (2006) señalan que es un rasgo que consta de cinco facetas diferentes: (1) Observar: la capacidad de atender experiencias internas y externas como percepciones, pensamientos, sensaciones o sentimientos; (2) Describir: la capacidad de describir experiencias internas a través de palabras ; (3) Actuar con conciencia: la capacidad de concentrarse en las actividades de uno/a en el momento; (4) No juzgar: la capacidad de no juzgar experiencias internas como pensamientos y sentimientos; y (5) No reaccionar: la capacidad de evitar dejarse llevar por la experiencia interna (Baer et al., 2006). Estas facetas pueden tener diferentes roles, dependiendo de la naturaleza del problema psicológico (Cortazar y Calvete, 2019), enfatizando la importancia de evaluar el rasgo de mindfulness a través de sus diferentes facetas. Sin embargo, una de las limitaciones para sacar conclusiones respecto al papel que tiene el MD en la protección frente a diferentes problemas psicológicos, es que muchos estudios previos se centran en solo una o algunas facetas del MD. Además, existen numerosas escalas para evaluar el constructo, algunas de las cuales son unidimensionales o se enfocan solo en algunas facetas del MD. No obstante, estudios anteriores indican que existe una superposición entre algunas facetas medidas con instrumentos diferentes. Por ejemplo, la escala de atención y conciencia plena (MAAS-A; Brown, West, Loverich y Biegel, 2011; versión española: Calvete, Sampedro y Orue, 2014) ha mostrado asociaciones con la faceta Actuar con conciencia del cuestionario de las cinco facetas de mindfulness (FFMQ; Quaglia, Braun, Freeman, McDaniel y Brown, 2016), y la Medida de Mindfulness en Niños y Adolescentes (CAMM; Greco, Baer y Smith, 2011; versión española: Guerra et al., 2019; Turanzas Romero, 2013) ha mostrado asociaciones con las facetas Actuar con conciencia y No juzgar del cuestionario FFMQ (Calvete y Royuela-Colomer, 2016).

A nivel transversal, algunos estudios encontraron que Actuar con conciencia se asoció con niveles más bajos de UPI (Gámez-Guadix y Calvete, 2016), como el uso compulsivo de teléfonos móviles y redes sociales (Apaolaza, Hartmann, D'Souza y Gilsanz, 2019; Kircaburun, Griffiths y Billieux, 2019). A nivel longitudinal, Calvete, Gámez-Guadix y Cortazar (2017a) encontraron que todas las facetas de MD (con la excepción de No reaccionar) predijeron niveles más bajos de UPI en adolescentes.

En cuanto al US, un metaanálisis indicó que, si bien muchos estudios mostraron relaciones negativas con MD, estos resultados son mixtos, ya que otros estudios no han encontrado estas relaciones o incluso encontraron relaciones positivas (Karyadi, VanderVeen y Cyders, 2014). Por ejemplo, en los y las adolescentes, las puntuaciones en el CAMM, que combina Actuar con conciencia y No juzgar, se asociaron con un menor consumo de alcohol y marihuana (Robinson, Ladd y Anderson, 2014), y Describir se asoció con un menor consumo de alcohol (Fernández, Wood, Stein y Rossi, 2010). Asimismo, los resultados de otro estudio transversal realizado con una muestra clínica adulta mostraron que estas tres facetas de MD se asociaron negativamente con US (Bowen y Enkema, 2014). Sin embargo, Karyadi et al. (2014) no encontraron asociaciones significativas para Observar o Describir, mientras que Actuar con conciencia, No juzgar y No reaccionar mostraron asociaciones negativas significativas. Además, la mayoría de los estudios existentes se han llevado a cabo bajo diseños transversales y muy pocos se han realizado con muestras de adolescentes. Un estudio reciente con una muestra de 
adolescentes no encontró predicciones significativas entre las puntuaciones en la escala MAAS (es decir, Actuar con conciencia) y el US (Calvete, Orue y Sampedro, 2017b).

El papel de la faceta Observar de MD ha sido objeto de debate. Aunque muchos estudios anteriores han encontrado que la faceta Observar puede ser desadaptativa en muestras de personas no meditadoras (p. ej., Baer et al., 2006; Calvete, Fernández-González, Echezarraga y Orue, 2019; Royuela-Colomer y Calvete, 2016), algunos estudios indicaron que esta faceta puede ser beneficiosa para diferentes problemas psicológicos al interactuar con otras habilidades de MD (p. ej., Desrosiers, Vine, Curtiss y Klemanski, 2014; Eisenlohr-Moul, Walsh, Charnigo, Lynam y Baer, 2012). En concreto, en una muestra de estudiantes universitarios, Eisenlohr-Moul et al. (2012) encontraron que Observar se asoció con menor US (es decir, tabaco y alcohol) solo en interacción con otras facetas, como No reaccionar. Por el contrario, Bowen y Enkema (2014) no encontraron un apoyo significativo para esta interacción. Además, que sepamos, no existen estudios que evalúen las interacciones entre Observar y otras facetas de MD para examinar los cambios en el UPI. Sin embargo, un metaanálisis reciente (Sala, Rochefort, Priscilla Lui y Baldwin, 2020) sugirió que Observar puede estar relacionada positivamente con conductas relacionadas con la salud cuando otras habilidades de MD son altas, lo que indica la necesidad de evaluar cómo la combinación de las facetas de MD puede influir en las conductas relacionadas con la salud.

\section{Descripción general del presente estudio}

Aunque estudios anteriores han evaluado la relación entre MD y conductas de riesgo como UPI y US, estos estudios, en general, utilizaron diseños transversales. Los estudios longitudinales son necesarios para evaluar hasta qué punto las facetas de MD predicen estos comportamientos de riesgo. Además, la mayoría de los estudios anteriores se han realizado con muestras adultas; sin embargo, como se mencionó anteriormente, estos comportamientos de riesgo tienden a surgir durante la adolescencia. Por último, la mayoría de los estudios existentes examinan el MD a través de una sola faceta o un constructo unidimensional, lo que dificulta determinar las relaciones específicas entre cada faceta y las conductas adictivas o las posibles interacciones entre Observar y las demás facetas.

Por tanto, el principal objetivo del presente estudio fue analizar si las cinco facetas del MD predecían niveles más bajos de UPI y US a lo largo del tiempo en una muestra de adolescentes. Teniendo en cuenta la revisión de la literatura, se esperaba que Describir, Actuar con Conciencia, No Juzgar y No Reaccionar predijeran menores niveles de UPI y US a lo largo del tiempo. Asimismo, el segundo objetivo fue evaluar las interacciones potenciales entre Observar y las demás facetas del constructo de MD en la predicción de los cambios en UPI y US. Se esperaba que la faceta Obser- var predijese menores niveles de UPI y US solo en combinación con niveles altos de otras facetas de MD, mitigando así el papel disfuncional que Observar puede tener en muestras no meditadoras.

\section{Método}

\section{Participantes}

Un total de 836 estudiantes de entre 11 y 18 años de edad $\left(M_{\text {edad }}=14,65\right.$ años, $\left.S D=1,74\right)$ completaron la línea base del presente estudio (423 chicas y 413 chicos). La distribución por edades fue: $11(7,4 \%), 12(17,2 \%), 13(9,9 \%), 14$ (16,5\%), 15 (24,4\%), $16(15,8 \%), 17(8 \%)$ y $18(0,7 \%)$. De esta muestra inicial, 650 estudiantes participaron en la segunda fase del estudio (tasa de retención $=77,75 \%$ ). Para el cálculo del nivel socioeconómico de los participantes, se siguieron los criterios sugeridos por la Sociedad Española de Epidemiología (2000): 13,6 \% bajo, 15,5\% medio-bajo, $29,1 \%$ medio, $16,2 \%$ medio-alto y $25,7 \%$ alto.

\section{Procedimiento}

Los y las participantes eran estudiantes de seis colegios seleccionados aleatoriamente de la lista completa de colegios públicos y privados de Álava y Vizcaya (España). Específicamente, participaron en el estudio dos escuelas públicas y cuatro privadas. Los y las estudiantes participaron voluntariamente con el consentimiento de sus madres, padres o tutores/as legales. Los equipos directivos de las escuelas fueron informados de los objetivos del estudio y tras recibir su aprobación, se les remitió información sobre los objetivos de la investigación y el correspondiente consentimiento informado a los y las estudiantes y sus madres y padres. Los y las estudiantes completaron los cuestionarios en sus aulas con una investigadora presente y sus respuestas fueron anónimas. Vinculamos sus respuestas en las dos fases del estudio (con seis meses de diferencia) utilizando un código que solo los y las participantes conocían. El Comité de Ética de la Universidad de Deusto aprobó este estudio.

\section{Mediciones}

Mindfulness disposicional. El MD se evaluó con la versión española del FFMQ adaptada a adolescentes (Baer et al., 2006; Royuela-Colomer y Calvete, 2016). Este cuestionario de autoinforme evalúa las cinco facetas de MD (es decir, Observar, Describir, Actuar con conciencia, No juzgar y No reaccionar) a través de 39 ítems. Los ítems se responden en una escala Likert desde 1 (nunca o muy raramente verdad) a 5 ( muy a menudo o siempre verdad). Algunos elementos, como muestra, son « Puedo poner fácilmente en palabras mis creencias, opiniones y las cosas que espero que vayan a pasar»; " Cuando hago algo mi mente tiende a divagar y distraerse»; $y$ « Percibo mis sentimientos y emociones sin tener que oponerme ante ellos». Estudios ante- 
riores han examinado las propiedades psicométricas de la escala FFMQ en muestras de niños, niñas y adolescentes (para revisión, ver Cortazar, Calvete, Fernández-González y Orue, 2020). En el presente estudio, los coeficientes $\alpha$ de Cronbach fueron ,75, ,75, ,82, ,86 y ,68, para Observar, Describir, Actuar con conciencia, No juzgar y No reaccionar, respectivamente.

Uso problemático de Internet. El UPI se evaluó con la Escala de Uso Problemático y Generalizado de Internet (GPIUS2; Caplan, 2010). El GPIUS2 es un cuestionario de autoinforme de 15 ítems que mide el uso generalizado y problemático de Internet. Los ítems se responden utilizando una escala Likert, desde 1 (totalmente en desacuerdo) a 6 (totalmente de acuerdo). Como ejemplo, algunos elementos son «Cuando no me conecto a Internet durante algún tiempo, empiezo a preocuparme con la idea de conectarme» $y$ «Tengo dificultad para controlar la cantidad de tiempo que estoy conectado/a a Internet». Este estudio utilizó las puntuaciones totales de UPI y la versión española del GPIUS2 (Gámez- Guadix, Orue y Calvete, 2013a). Las propiedades psicométricas del GPIUS2 resultaron adecuadas tanto en la versión original como en la española (Caplan, 2010; Gámez-Guadix et al., 2013a). En este estudio, los coeficientes $\alpha$ de Cronbach para la puntuación total fueron ,92 en el Tiempo 1 (T1) y ,91 en el Tiempo 2 (T2).

Consumo de sustancias. El US se evaluó con el Inventario de consumo de drogas en adolescentes (Calvete y Estévez, 2009). Esta escala de autoinforme comprende nueve ítems sobre la frecuencia del consumo de diferentes sustancias. En este estudio se evaluó la frecuencia de consumo de alcohol, marihuana, hachís, cocaína, «speed» y éxtasis. Los ítems se responden usando una escala Likert desde 0 (nunca) a 5 (diariamente). En este estudio, los coeficientes $\alpha$ de Cronbach para la puntuación total fueron ,64 en T1 y ,65 en T2.

\section{Análisis de datos}

La prueba MCAR de Little indicó que los valores perdidos no eran aleatorios, $\chi^{2}(103)=218, p<, 000$. Aquellos/ as que solo participaron en la primera fase obtuvieron puntuaciones más bajas en Actuar con conciencia $(t=-2,81$, $p=, 005, d=-, 23)$ y No juzgar $(t=-3,31, p=, 001, d=-, 27)$ y más altas en UPI $(t=2,59, p=, 01, d=, 23)$, US $(t=6,17$, $p=, 000, d=, 63)$, y edad $(t=8,99, p=, 000, d=, 75)$. Por tanto, para gestionar los valores perdidos, utilizamos el método de máxima verosimilitud con información completa (FIML) con MPLUS 8. El modelo hipotetizado incluyó: (1) asociaciones transversales entre todas las variables del estudio en T1 y T2, (2) trayectorias autorregresivas de las variables en T1 a las mismas variables en T2 (UPI y US), (3) trayectorias predictivas cruzadas desde las dimensiones de MD de T1 a UPI y US de T2, y (4) trayectorias predictivas de los términos de interacción entre Observar y las otras cuatro dimensiones de MD (es decir, Actuar con conciencia x Observar, No juzgar x Observar, Describir x Observar y No reaccionar x Observar). Siguiendo el procedimiento estándar para examinar los efectos de la moderación, todas las dimensiones de MD se transformaron en puntuaciones $z$ en T1. Las figuras de interacción fueron creadas utilizando una macro de Dawson (2018).

La bondad de ajuste del modelo se evaluó utilizando el índice de ajuste comparativo (CFI), el índice de ajuste no normativo (NNFI), el error cuadrático medio de aproximación (RMSEA) y la raíz cuadrada media residual estandarizada (SRMR). En general, los valores de CFI y NNFI iguales o superiores a ,90 reflejan un buen ajuste, y los valores de RMSEA y SRMR inferiores a ,08 indican un ajuste excelente (Hu y Bentler, 1999).

La aplicación desarrollada por Preacher y Coffman (2006) se utilizó para realizar un análisis de potencia, es decir, para calcular la probabilidad de detectar un efecto, en caso de haber presente un efecto real para detectar. La potencia en este estudio fue del 99,9\% para una muestra de 836 participantes. Todos los datos están disponibles en Open Science Framework (https://osf.io/p2967/).

\section{Resultados}

\section{Estadísticos descriptivos y análisis correlacional}

La Tabla 1 muestra los estadísticos descriptivos y los coeficientes de correlación entre todas las variables del estudio. En general, las dimensiones de MD se asociaron negativamente con UPI y US en T1 y T2. Específicamente, Actuar con conciencia y No juzgar se asociaron negativamente tanto con UPI como con US en ambos puntos temporales, mientras que Describir se asoció negativamente solo con UPI en T1. UPI y US se asociaron positivamente entre sí en ambos puntos temporales.

\section{Modelo predictivo}

El modelo predictivo a través de path análisis mostró índices de ajuste excelentes $c^{2}(36, N=836)=1085,063$, RM$\mathrm{SEA}=, 052$ (90\% IC [,034-,072]), SRMR = ,02, NNFI = ,92, $\mathrm{CFI}=, 98$. El modelo explicó el $42 \%$ de la varianza de UPI en T2 y el $50 \%$ de la varianza de US en T2.

La Figura 1 muestra las trayectorias longitudinales significativas y marginalmente significativas del modelo. Las trayectorias autorregresivas para UPI y US fueron estadísticamente significativas, indicativo de la alta estabilidad de ambas variables en el tiempo. Con respecto a las trayectorias predictivas desde las dimensiones de MD en T1 hacia las medidas de conductas adictivas en T2, Actuar con conciencia predijo menores niveles de US, y No juzgar predijo marginalmente menores niveles de UPI; sin embargo, Describir predijo un aumento tanto en UPI como en US. Además, el término de interacción entre Actuar con conciencia y Observar predijo cambios en UPI.

La Figura 2 muestra la forma de esta interacción para adolescentes que puntuaron bajo $(z=-1)$ y alto $(z=1)$ en 
Tabla 1. Coeficientes de correlación entre variables y estadísticas descriptivas.

\begin{tabular}{|c|c|c|c|c|c|c|c|c|c|c|}
\hline & 1 & 2 & 3 & 4 & 5 & 6 & 7 & 8 & 9 & 10 \\
\hline \multicolumn{11}{|l|}{ 1. T1 UPI } \\
\hline 2. T1 US &, $15^{* *}$ & & & & & & & & & \\
\hline 3. T1 0 &, 04 &, 04 & & & & & & & & \\
\hline 4. T1 D &,$- 10^{\star \star}$ &,- 03 &, $22^{* *}$ & & & & & & & \\
\hline 5. T1 AC &,$- 37^{\star \star}$ &,$- 25^{* *}$ &,$- 10^{\star \star}$ &, $25^{* \star}$ & & & & & & \\
\hline 6. T1 NJ &,$- 34^{* \star}$ &,$- 10^{* *}$ &,$- 27^{\star \star}$ &, $17^{* *}$ &, $40^{* *}$ & & & & & \\
\hline 7. T1 NR &,- 02 &, 04 &, $41^{* *}$ &, $37^{* \star}$ &,- 04 &,$- 10^{* *}$ & & & & \\
\hline 8. T2 UPI &, $63^{* *}$ &, $16^{\star \star}$ & ,02 &,- 03 &,$- 27^{\star \star}$ &,$- 27^{* *}$ &,- 01 & & & \\
\hline 9. T2 US &, $13^{* *}$ &, $65^{\star *}$ &, 05 &, 03 &,$- 21^{\star \star}$ &,$- 09^{\star}$ &, 02 &, $16^{\star \star}$ & & \\
\hline 10. Edad &, $30^{* *}$ &, $52^{\star \star}$ &, $07^{*}$ &,- 02 &,$- 34^{\star \star}$ &,$- 19^{\star \star}$ &, 02 &, $30^{* *}$ &, $44^{\star \star}$ & \\
\hline Media & 1,90 & 0,32 & 2,81 & 3,16 & 3,50 & 3,67 & 2,71 & 1,90 & 0,30 & 14,65 \\
\hline SD & 0,91 & 0,47 & 0,74 & 0,67 & 0,75 & 0,82 & 0,65 & 0,87 & 0,49 & 1,74 \\
\hline
\end{tabular}

Nota. $\mathrm{T} 1$ = Tiempo $1 ; \mathrm{T} 2$ = Tiempo 2; UPI = Uso problemático de Internet; US = Uso de sustancias; $0=$ Observar; $\mathrm{D}=$ Describir; $\mathrm{AC}=\mathrm{Actuar}$ con conciencia; $\mathrm{NJ}=$ No juzgar; NR $=$ No reaccionar. ${ }^{*} \mathrm{p}<, 05 .{ }^{* \star} \mathrm{p}<, 01$.

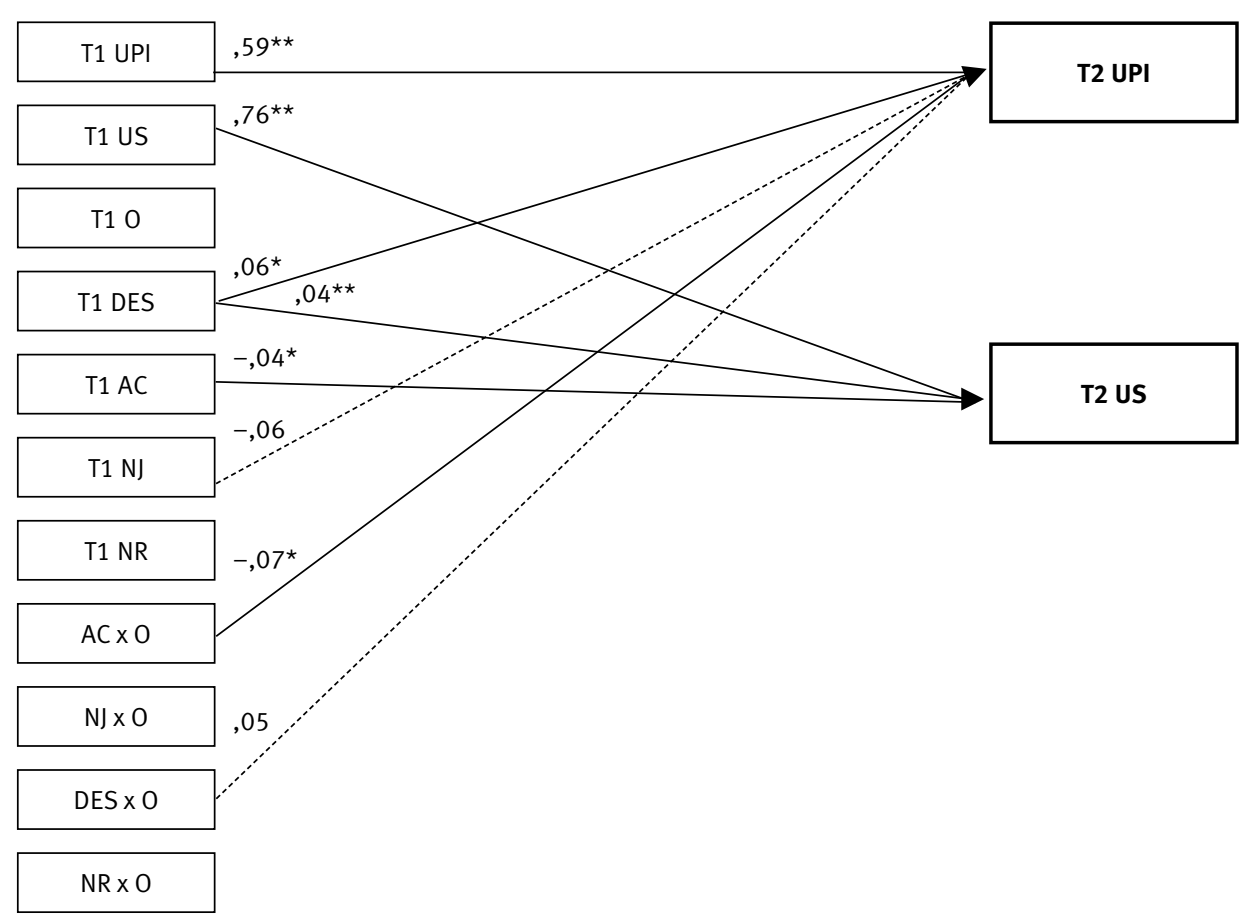

Figura 1. Trayectorias longitudinales estadísticamente significativas del modelo general.

Nota. $\mathrm{T} 1$ = Tiempo 1; T2 = Tiempo 2; UPI = Uso problemático de Internet; US = Uso de sustancias; AC = Actuar con conciencia; $\mathrm{NJ}=$ No juzgar; $\mathrm{NR}=$ No reaccionar; DES = Describir; $0=$ Observar. Los valores proporcionados son coeficientes estandarizados. $p<, 05 .{ }^{\star \star} p<, 01$. ${ }^{\star * *} p<, 001$. Las líneas discontinuas representan trayectorias marginalmente significativas $(p=, 07)$.

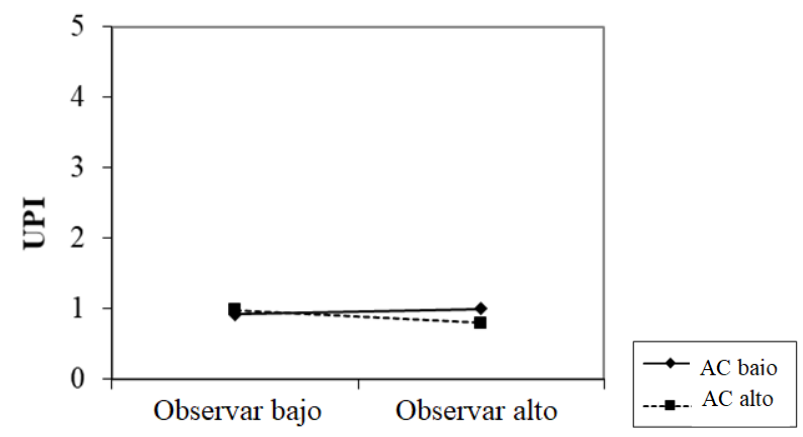

Figura 2. Interacción entre Actuar con conciencia y Observar para UPI. Nota. UPI = Uso problemático de Internet; $\mathrm{AC}=$ Actuar con conciencia.

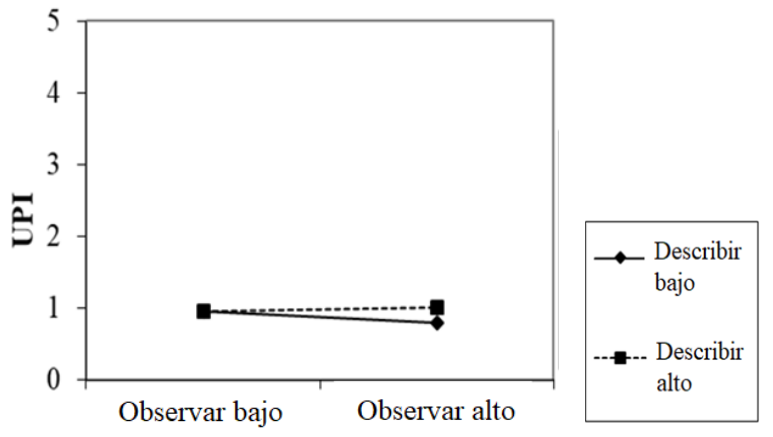

Figura 3. Interacción entre Describir y Observar para UPI. Nota. UPI = Uso problemático de Internet. 
las dimensiones Actuar con conciencia y Observar. La asociación predictiva entre T1 Observar y T2 UPI es negativa cuando Actuar con conciencia es alta. Por último, el término de interacción entre Describir y Observar predijo marginalmente cambios en UPI. La Figura 3 muestra la forma de esta interacción, para adolescentes que puntuaron bajo $(z=-1)$ y alto $(z=1)$ en estas dimensiones. Los hallazgos indicaron que Observar podía predecir menos UPI solo cuando Describir era bajo.

\section{Discusión}

El presente estudio evaluó longitudinalmente el papel que tienen las facetas diferenciadas de MD en la protección frente a UPI y US. Asimismo, teniendo en cuenta el papel desadaptativo de la faceta Observar frente a numerosos problemas, se evaluaron las interacciones entre Observar y las demás facetas de MD para predecir cambios en UPI y US.

Los resultados transversales, de acuerdo con estudios previos (p. ej., Kircaburun et al., 2019; Robinson et al., 2014), indicaron asociaciones negativas entre la mayoría de las facetas de MD (es decir, Actuar con conciencia, No juzgar y Describir) y ambas conductas de riesgo (US y UPI). A nivel longitudinal, Actuar con conciencia predijo niveles más bajos de US y No juzgar predijo marginalmente niveles más bajos de UPI a lo largo del tiempo, en coherencia con los resultados de estudios anteriores (p. ej., Calvete et al., 2017a; Robinson et al., 2014). Se ha sugerido que los y las adolescentes que son más capaces de no juzgar sus experiencias internas y que pueden actuar con conciencia, pueden ser más propensos/as a aceptar sus emociones negativas y darse cuenta cuando no se comportan de manera saludable (Sala et al., 2020). Por tanto, es menos probable que estos/as adolescentes intenten aliviar su angustia emocional mediante el US o el UPI. Contrario a estudios anteriores (p. ej., Bowen y Enkema, 2014; Calvete et al., 2017a), Describir predijo mayores niveles tanto de UPI como de US. Además, Describir pareció tener un papel desadaptativo para UPI, especialmente en combinación con altos niveles de Observar. Hay varias explicaciones tentativas para estos hallazgos. Se ha sugerido que describir y etiquetar con palabras lo que uno o una siente, si no se hace correctamente, puede conducir a una menor participación en conductas que promueven la salud (Sala et al., 2020). Además, puede ser que los y las adolescentes que más observan y describen sus emociones sean los/as que experimentan las emociones más negativas, por lo que la asociación entre emociones negativas y conductas adictivas (Kassel et al., 2007) podría explicar este resultado.

En este estudio, Observar no resultó ser un predictor significativo de US o UPI. Este hallazgo fue coherente con los resultados obtenidos en el metaanálisis realizado por Karyadi et al. (2014), que indicó que Observar no predecía el US; sin embargo, eso contradice los resultados obteni- dos por Calvete et al. (2017a), quienes encontraron que Observar desempeñaba un papel adaptativo en relación con el UPI. Esta diferencia podría deberse a que Calvete et al. (2017a) solo examinaron las trayectorias directas entre las facetas de MD y UPI, mientras que el presente estudio incluyó los términos de interacción entre Observar y las demás facetas. De hecho, nuestros resultados son consistentes con la literatura previa que destaca que esta faceta puede ser beneficiosa en la interacción con otras facetas de MD (Desrosiers et al., 2014; Eisenlohr-Moul et al., 2012). En este estudio, Observar fue beneficiosa frente al UPI cuando se combinó con altos niveles de Actuar con conciencia. Muchos estudios han demostrado cómo la faceta Observar es más adaptativa en muestras de personas que practican meditación (p. ej., Baer et al., 2006; 2008), lo que indica que pueden ser más capaces que otras personas para actuar con conciencia, no juzgar las experiencias internas y no reaccionar con impulsividad. Por tanto, parece que Observar puede proteger a los y las adolescentes frente al UPI solo cuando también tienen la capacidad de actuar con conciencia. De hecho, estos resultados son coherentes con la idea de que aquellos que obtuvieron puntuaciones altas en Observar deben intentar desarrollar la faceta Actuar con conciencia (Sala et al., 2020).

\section{Limitaciones e investigación futura}

El presente estudio tiene algunas limitaciones. Primero, todas las evaluaciones se llevaron a cabo utilizando medidas de autoinforme. La investigación futura podría incluir otras fuentes de información, tales como informes parentales y/o de pares. Segundo, las medidas de No reaccionar y US mostraron baja confiabilidad, lo que puede estar condicionando los resultados obtenidos respecto a estas variables. En el caso de US, la baja consistencia puede deberse a que el uso de una sustancia en particular no tiene por qué estar asociado con el uso de otras sustancias. Tercero, aunque este estudio incluyó una muestra grande de adolescentes, el rango de edad fue amplio. Sería interesante para estudios futuros evaluar si los hallazgos de este estudio se mantienen o difieren en diferentes rangos de edad. Por último, a pesar de ser un estudio longitudinal, los estudios futuros podrían incluir más puntos temporales o un intervalo más largo entre mediciones, para observar si los resultados se mantienen en el tiempo.

A pesar de sus limitaciones, este estudio también tiene varias fortalezas, como es su diseño longitudinal. Que sepamos, la mayor parte de la investigación existente en este campo ha sido transversal; por tanto, es importante realizar estudios que permitan evaluar cómo las facetas de MD protegen contra el US y el UPI a lo largo del tiempo. Además, este estudio se realizó con una muestra grande de adolescentes. Teniendo en cuenta que muchas conductas adictivas que persisten en la adultez comienzan durante la adolescencia, es importante contar con más investiga- 
ciones que nos permitan determinar factores protectores para los y las adolescentes frente a problemas cada vez más frecuentes, como UPI y US.

\section{Conclusión}

Este estudio indica que no todas las facetas de la MD son beneficiosas frente al UPI y el US en adolescentes. Específicamente, parece que las facetas No juzgar y Actuar con conciencia pueden jugar un papel relevante para los y las adolescentes al enfrentar estos problemas. Además, Actuar con conciencia parece proteger a aquellos y aquellas adolescentes con altos niveles de Observar. En cuanto a Describir, sería interesante para futuros estudios analizar en detalle el papel de esta faceta en combinación con otras variables, como los niveles de estrés y angustia emocional.

En general, los hallazgos de este estudio pueden proporcionar información útil para el desarrollo de nuevas intervenciones basadas en mindfulness dirigidas a prevenir conductas adictivas en adolescentes. Las técnicas encaminadas a mejorar las diferentes dimensiones de DM deben depender de la conducta adictiva a prevenir. Por tanto, si otros estudios confirman los resultados del presente estudio, las intervenciones dirigidas a reducir el US podrían incluir técnicas para mejorar la capacidad de actuar con conciencia, mientras que las destinadas a reducir el UPI deberían mejorar la capacidad de los y las adolescentes de no juzgar sus experiencias internas.

\section{Reconocimientos}

Este trabajo recibió una subvención del Ministerio de Economía y Competitividad (Gobierno de España, Ref. PSI2015-68426-R), del País Vasco (Ref. IT982-16 y Ref. PI_2016_1_0023), y de BBVA (PR[18]_SOC_0096). Ninguno participó en el diseño del estudio, la recopilación, el análisis o la interpretación de los datos, la redacción del manuscrito o la decisión de remitir el artículo para su publicación.

\section{Conflicto de intereses}

Las autoras declaran la inexistencia de conflicto de interés.

\section{Referencias}

Apaolaza, V., Hartmann, P., D’Souza, C. y Gilsanz, A. (2019). Mindfulness, compulsive mobile social media use, and derived stress: The mediating roles of self-esteem and social anxiety. Cyberpsychology, Behavior and Social Networking, 22, 388-396. doi:10.1089/cyber.2018.0681.

Baer, R. A., Smith G. T., Hopkins, J., Krietemeyer, J. y Toney, L. (2006). Using self-report assessment methods to explore facets of mindfulness. Assessment, 13, 27-45. doi:10.1177/1073191105283504.

Baer, R. A., Smith, G. T., Lykins, E., Button, D., Krietemeyer, J., Sauer, S.,...Williams, J. M. G. (2008). Construct validity of the five facet mindfulness questionnaire in meditating and nonmeditating samples. Assessment, 15, 329-342. doi:10.1177/1073191107313003.

Bava, S. y Tapert, S. F. (2010). Adolescent brain development and the risk for alcohol and other drug problems. Neuropsychology Review, 20, 398-413. doi:10.1007/s11065010-9146-6.

Bishop, S. R., Lau, M., Shapiro, S., Carlson, L., Anderson, N. D., Carmody, J.,... Devins, G. (2004). Mindfulness: A proposed operational definition. Clinical Psychology: Science and Practice, 11, 230-241. doi:10.1093/clipsy/ bph077.

Bowen, S. y Enkema, M. C. (2014). Relationship between dispositional mindfulness and substance use: Findings from a clinical sample. Addictive Behaviors, 39, 532-537. doi:10.1016/j.addbeh.2013.10.026.

Brown, K., West, A., Loverich, T. y Biegel, G. (2011). Assessing adolescent mindfulness: Validation of an Adapted Mindful Attention Awareness Scale in adolescent normative and psychiatric populations. Psychological Assessment, 23, 1023-1033. doi:10.1037/a0021338.

Brownlie, E., Beitchman, J. H., Chaim, G., Wolfe, D. A., Rush, B. y Henderson, J. (2019). Early adolescent substance use and mental health problems and service utilisation in a school-based sample. The Canadian Journal of Psychiatry, 64, 116-125. doi:10.1177/0706743718784935.

Calvete, E. y Estévez, A. (2009). Substance use in adolescents: The role of stress, impulsivity, and schemas related to lack of limits. Adicciones, 21, 49-56. doi:10.20882/ adicciones.251.

Calvete, E., Fernández-González, L., Echezarraga, A. y Orue, I. (2019). Dispositional mindfulness profiles in adolescents and their associations with psychological functioning and hypothalamic-pituitary-adrenal axis hormones. Journal of Youth and Adolescence, 1-14. doi:10.1007/s10964-019-01128-6.

Calvete, E., Gámez-Guadix, M. y Cortazar, N. (2017a). Mindfulness facets and problematic Internet use: A six-month longitudinal study. Addictive Behaviors, 72, 57-63. doi:10.1016/j.addbeh.2017.03.018.

Calvete, E., Orue, I. y Sampedro, A. (2017b). Does the acting with awareness trait of mindfulness buffer the predictive association between stressors and psychological symptoms in adolescents? Personality and Individual Differences, 105, 158-163. doi:10.1016/j.paid.2016.09.055.

Calvete, E. y Royuela-Colomer, E. (2016). Measurement of dispositional mindfulness in children and adolescents: A review of available self-report measures in Spanish. Mindfulness $\mathcal{E}$ Compassion, 1, 58-67. doi:10.1016/j.mincom.2016.11.001. 
Calvete, E., Sampedro, A. y Orue, I. (2014). Propiedades psicométricas de la versión española de la "Escala de atención y conciencia plena para adolescentes" (Mindful Attention Awareness Scale - Adolescents) (MAAS-A). Psicología Conductual, 22, 277-291.

Caplan, S. E. (2010). Theory and measurement of generalized problematic Internet use: A two-step approach. Computers in Human Behavior, 26, 1089-1097. doi:10.1016/j. chb.2010.03.012.

Cortazar, N. y Calvete, E. (2019). Dispositional mindfulness and its moderating role in the predictive association between stressors and psychological symptoms in adolescents. Mindfulness, 10, 2046-2059. doi:10.1007/s12671019-01175-x.

Cortazar, N., Calvete, E., Fernández-González, L. y Orue, I. (2020). Development of a short form of the Five Facet Mindfulness Questionnaire -Adolescents (FFMQ-A) for children and adolescents. Journal of Personality Assessment, 102, 641-652. doi:10.1080/00223891.2019.1616206 .

Dawson, J. F. (2018). Interpreting Interaction Effects. Recuperado de http://www.jeremydawson.com/slopes.htm.

Desrosiers, A., Vine, V., Curtiss, J. y Klemanski, D. H. (2014). Observing nonreactively: A conditional process model linking mindfulness facets, cognitive emotion regulation strategies, and depression and anxiety symptoms. Journal of Affective Disorders, 165, 31-37. doi:10.1016/j. jad.2014.04.024.

Eisenlohr-Moul, T. A., Walsh, E. C., Charnigo, R. J., Lynam, D. R. y Baer, R. A. (2012). The "what" and the "how" of dispositional mindfulness. Assessment, 19, 276-286. doi:10.1177/1073191112446658.

Fernández, A. C., Wood, M. D., Stein, L. A. R. y Rossi, J. S. (2010). Measuring mindfulness and examining its relationship with alcohol use and negative consequences. Psychology of Addictive Behaviors, 24, 608-616. doi:10.1037/ a0021742.

Gámez-Guadix, M. y Calvete, E. (2016). Assessing the relationship between mindful awareness and problematic Internet use among adolescents. Mindfulness, 7, 12811288. doi:10.1007/s12671-016-0566-0.

Gámez-Guadix, M., Orue, I. y Calvete, E. (2013a). Evaluation of the cognitive-behavioral model of generalized and problematic Internet use in Spanish adolescents. Psicothema, 25, 299-306. doi:10.7334/psicothema2012.274.

Gámez-Guadix, M., Orue, I., Smith, P. K. y Calvete, E. (2013b). Longitudinal and reciprocal relations of cyberbullying with depression, substance use, and problematic internet use among adolescents. Journal of Adolescent Health, 53, 446-452. doi:10.1016/j.jadohealth.2013.03.030.

Gómez, P., Rial, A., Braña, T., Golpe, S. y Varela, J. (2017). Screening of Problematic Internet Use among Spanish adolescents: Prevalence and related variables. Cy- berpsychology, Behavior and Social Networking, 20, 259-267. doi:10.1089/cyber.2016.0262.

Greco, L. A., Baer, R. A. y Smith, G. T. (2011). Assessing mindfulness in children and adolescents: Development and validation of the Child and Adolescent Mindfulness Measure (CAMM). Psychological Assessment, 23, 606-614. doi:10.1037/ a0022819.

Guerra, J., García-Gómez, M., Turanzas, J., Cordón, J. R., Suárez-Jurado, C. y Mestre, J. M. (2019). A brief Spanish version of the Child and Adolescent Mindfulness Measure (CAMM). A dispositional mindfulness measure. International Journal of Environmental Research and Public Health, 16, 1355-1367. doi:10.3390/ijerph16081355.

$\mathrm{Hu}$, L. T. y Bentler, P. M. (1999). Cutoff criteria for fit indexes in covariance structure analysis: Conventional criteria versus new alternatives. Structural Equation Modeling: A Multidisciplinary Journal, 6, 1-55. doi:10.1080/10705519909540118.

Karyadi, K. A., VanderVeen, J. D. y Cyders, M. A. (2014). A meta-analysis of the relationship between trait mindfulness and substance use behaviors. Drug and Alcohol Dependence, 143, 1-10. doi:10.1016/j.drugalcdep.2014.07.014.

Kassel, J. D., Greenstein, J. E., Evatt, D. P., Roesch, L. L., Veilleux, J. C., Wardle, M. C. y Yates, M. C. (2007). Negative affect and addiction. En M. Al'Absi (Ed.), Stress and Addiction: Biological and Psychological Mechanisms (pp. 171-189). Amsterdam: Elsevier Academic Press.

Kircaburun, K., Griffiths, M. D. y Billieux, J. (2019). Trait emotional intelligence and problematic online behaviors among adolescents: The mediating role of mindfulness, rumination, and depression. Personality and Individual Differences, 139, 208-213. doi:10.1016/j.paid.2018.11.024.

Observatorio Español de las Drogas y las Adicciones (2019). Encuesta sobre Uso de Drogas en Enseñanzas Secundarias en España. Recuperado de http://www.pnsd.mscbs. gob.es/profesionales/sistemasInformacion/sistemaInformacion/pdf/ESTUDES_2018-19_Informe.pdf.

Preacher, K. J. y Coffman, D. L. (2006, May). Computing power and minimum sample size for RMSEA [Computer software]. Recuperado de http:/ / quantpsy.org/.

Quaglia, J. T., Braun, S. E., Freeman, S. P., McDaniel, M. A. y Brown, K. W. (2016). Meta-analytic evidence for effects of mindfulness training on dimensions of self-reported dispositional mindfulness. Psychological Assessment, 28, 803-818. doi:10.1037/pas0000268.

Restrepo, A., Scheininger, T., Clucas, J., Alexander, L., Salum, G., Georgiades, K.,... Milham, M. (2020). Problematic Internet Use in children and adolescents: Associations with psychiatric disorders and impairment. $B M C$ Psychiatry, 20, 1-11. doi:10.1186/s12888-020-02640-x 7.

Robinson, J. M., Ladd, B. O. y Anderson, K. G. (2014). When you see it, let it be: Urgency, mindfulness and adolescent substance use. Addictive Behaviors, 39, 10381041. doi:10.1016/j.addbeh.2014.02.011. 
Royuela-Colomer, E. y Calvete, E. (2016). Mindfulness facets and depression in adolescents: Rumination as a mediator. Mindfulness, 7, 1092-1102. doi:10.1007/s12671016-0547-3.

Sala, M., Rochefort, C., Priscilla Lui, P. y Baldwin, A. S. (2020). Trait mindfulness and health behaviors: A meta-analysis. Health Psychology Review, 14, 345-393. doi:10.1 080/17437199.2019.1650290.

Sociedad Española de Epidemiología y la Sociedad Española de Medicina de Familia y Comunitaria (2000). A proposed measure of social class. Atención Primaria, 25, 350-363. doi:10.1016/S0212-6567(00)78518-0.

Turanzas Romero, J. (2013). Adaptación transcultural de la escala CAMM (Child and Adolescent Mindfulness Measure) y estudio preliminar de sus características psicométricas. Master's Degree Final Project. Máster en Terapias Psicológicas de $3^{\text {a }}$ Generación Orientación Investigadora. Valencia: Valencian International University. doi:10.13140/ RG.2.2.23873.35681. 
